\title{
On A Simple Econometric Approach for Utility-Based Asset Pricing Model
}

\author{
Jack C. Lee, National Chiao-Tung University \\ Cheng-Few Lee*, Rutgers University and National Chiao-Tung University \\ H.F. Ni, National Chiao-Tung University
}

\begin{abstract}
The Journal of Finance has published an important paper entitled "A Simple Econometric Approach for Utility-Based Asset Pricing Model" by Brown and Gibbon (1985). The main purpose of this paper is to extend the research of Brown and Gibbons (1985) and Karson et al. (1995) in estimating the relative risk aversion (RRA) parameter $\beta$ in utility-based asset pricing model. First, we review the distributions of RRA parameter estimate $\hat{\beta}$. Then, a new method to the distribution of $\hat{\beta}$ is derived, and a Bayesian approach for the inference of $\beta$ is proposed. Finally, empirical results are presented by using market rate of return and riskless rate data during the period December 1925 through December 1999.
\end{abstract}

* Corresponding author: Professor Cheng-Few Lee, Department of Finance, School of Business, Rutgers University, Piscataway, NJ 08854-8054, U.S.A. Phone: (732) 445-3530; Fax: (732) 445-5927; E-mail: Lee@ @rbs.rutgers.edu 
February 2002 version

\section{A. Introduction}

Brown and Gibbons (1985) and Karson, Cheng, and Lee (1995) have proposed different methods for estimating the relative risk aversion parameter. This paper first proposes a new approach to deal with the statistical distribution of the relative risk aversion estimator derived by Karson, Cheng, and Lee. In addition, a Bayesian statistical methodology is used to construct the interval estimation for the relative risk aversion. Furthermore, it also examines the statistical distribution of excess market rate of return in accordance with Box and Cox (1964) transformation to determine whether the lognormal distribution is suitable for the data at hand in estimating the relative risk aversion.

In section $\mathrm{B}$, an exact distribution for parametric estimation of the relative risk aversion (RRA) is examined in detail. In section $C$ an alternative method to the distribution of $\hat{\beta}$ is explored. Section D proposed a Bayesian approach for the inference of $\beta$. Empirical results are presented in section E. Finally, section F summarized the results of the paper.

\section{B. A brief literature review of RRA Estimation}

Let $R_{\mathrm{M}}$ be the market rate of return, $R_{\mathrm{f}}$ be the riskless rate of return, $X=\left(1+R_{\mathrm{M}}\right) /\left(1+R_{\mathrm{f}}\right)$ and $Y=\log X$. Furthermore, let $\left\{R_{\mathrm{Mt}}\right\}$ and $\left\{R_{\mathrm{ft}}\right\}, \mathrm{t}=1, \ldots, T$, be the observed samples. Then the sample mean and the sample variance of excess market rate of return are

$$
\bar{Y}=\overline{\log X}=\frac{\sum_{\mathrm{t}=1}^{\mathrm{T}} \mathrm{Y}_{\mathrm{t}}}{\mathrm{T}},
$$

and 
$S^{2}=\frac{\sum_{\mathrm{t}=1}^{\mathrm{T}}\left(\mathrm{Y}_{\mathrm{t}}-\bar{Y}\right)^{2}}{\mathrm{~T}-1}$.

Assuming normality for $Y$ with mean $\mu$ and variance $\sigma^{2}$, Brown and Gibbons (1985)

established the following relative risk aversion $(R R A)$,

$\beta=\frac{\mu}{\sigma^{2}}+\frac{1}{2}$

Following Brown and Gibbons, a natural maximum likelihood estimator for $\beta$ is

$\hat{b}=\frac{\bar{Y}}{S^{2}}+\frac{1}{2}$

Using asymptotic theory, Brown and Gibbons have derived the variance of $\sqrt{T} \hat{b}$ as:

$\operatorname{Var}\{\sqrt{T} \hat{b}\}=\frac{2\left[E\left\{\ln \tilde{x}_{t}\right\}\right]^{2}+\operatorname{Var}\left\{\ln \tilde{x}_{t}\right\}}{\left[\operatorname{Var}\left\{\ln \tilde{x}_{t}\right\}\right]^{2}}$.

Alternatively, following Karson et al. (1995), the minimum variance unbiased (MVU)

estimator of $\beta$ is

$\hat{\beta}=\frac{(T-3) \bar{Y}}{(T-1) S^{2}}+\frac{1}{2}$

In case the normality assumption for $Y$ is violated, the estimator $\hat{b}$ can be inconsistent, as pointed out by Brown and Gibbons. In order to remedy this possible shortcoming, they proposed a method of moment estimator which is the solution of

$f(b)=\frac{1}{T} \sum_{t=1}^{T}\left(X_{t}-1\right) X_{t}^{-b}=0$,

with the asymptotic variance

$\operatorname{Var}(\sqrt{T} b)=\frac{E\left\{\left[\left(X_{t}-1\right) X_{t}^{-\beta}\right]^{2}\right\}}{\left[E\left\{\left(X_{t}-1\right) X_{t}^{-\beta} \log X_{t}\right]^{2}\right.}$,

where $\beta$ is the relative risk aversion. 
Karson et al. (1995) have derived the exact distribution of $\hat{\beta}$, which is defined in Equation (6), as:

$$
f(\hat{\beta})=\frac{c_{1}}{v} \sum_{r=0}^{\infty} \Gamma\left(\frac{2 r+T+1}{4}\right) \frac{1}{r !} \cdot\left(\frac{2 T \sigma^{2}(\beta-1 / 2)(\hat{\beta}-1 / 2)((T-1) /(T-3)-(T-1)}{\sqrt{2 T} \sigma(\hat{\beta}-1 / 2)((T-1) /(T-3))}\right)^{r},
$$

where

$$
\begin{aligned}
& c_{1}=\frac{e^{-T(\beta-1 / 2)^{2} \sigma^{2} / 2}}{\sqrt{\pi} 2^{\frac{T+3}{4}}\left(\frac{\sigma \sqrt{T}}{T-1}\right)^{\frac{T-1}{2}} \Gamma\left(\frac{T-1}{2}\right)} \frac{(T-1)}{(T-3)}, \\
& v=\left[(\hat{\beta}-1 / 2)\left(\frac{T-1}{T-3}\right)\right]^{\frac{T+1}{2}} .
\end{aligned}
$$

The exact distribution presented in the above equation is expressed in terms of an infinite sum, therefore, it is not easy to compute in practice.

\section{A new method to the distribution of $\hat{\beta}$}

The exact distribution of $\hat{\beta}$ obtained by Karson et al. (1995) as given in Equation (9) is not easy to compute in practice. We will next propose a new method to the distribution of $\hat{\beta}$. We first note that the relative risk aversion estimator $\hat{\beta}$, as defined in Equation (6), can be rewritten as:

$$
\hat{\beta}=\frac{(T-3) \bar{Y}}{(T-1) S^{2}}+\frac{1}{2}=\frac{(T-3) \bar{Y} / \sigma^{2}}{(T-1) S^{2} / \sigma^{2}}+\frac{1}{2}=\frac{(T-3) \bar{Y} / \sigma^{2}}{W}+\frac{1}{2},
$$

where $\bar{Y}$ and $W=(T-1) S^{2} / \sigma^{2}$ are independent, and $\bar{Y} \sim \mathrm{N}\left(\mu, s^{2} / \mathrm{T}\right), \mathrm{W} \sim \chi_{T-1}^{2}$.

It's easy to show that

$\mathrm{E}(\hat{\beta})=\beta$, 
and

$\sigma_{\hat{\beta}}^{2}=\mathrm{V}(\hat{\beta})=\frac{T-3}{(T-5) \sigma^{2}}\left[\frac{1}{T}+\frac{2 \mu^{2}}{(T-3) \sigma^{2}}\right]$

as given in Karson et al.

From Equation (12) we can express the distribution of $\hat{\beta}$ as

$$
f(\hat{\beta})=\int_{0}^{\infty} f(\hat{\beta} \mid w) g(w) d w
$$

where $f(\hat{\beta} \mid w)$ is the p.d.f. of normal distribution with mean $\frac{(T-3) \mu}{\sigma w}+\frac{1}{2}$, variance $\frac{(T-3)^{2}}{w^{2} T \sigma^{2}}$, and $g(w)$ is the p.d.f. of $\chi_{T-1}^{2}$

The distribution of $\hat{\beta}$ given in Equation (15) is a one-dimensional integral. We will next consider two approximations:

$f(\hat{\beta}) \approx f(\hat{\beta} \mid \hat{w})$

where $\hat{w}$ is the mode of $\chi_{T-1}^{2}$, which is $T-3$. Following Ljung and Box (1980), this approximation will be reasonable if $g(w)$ is symmetric and concentrated. This will be the case when $T$ is reasonably large. Under this approxima tion, $\hat{\beta}$ is normally distributed as indicated in Equation (15) and with $\hat{w}=T-3$ and $\sigma^{2}=\frac{(T-1)}{(T-3)} S^{2}$.

A better approximation is:

$$
f(\hat{\beta}) \approx \frac{1}{L} \sum_{i=1}^{L} f\left(\hat{\beta} \mid w^{(i)}\right)
$$


where $w^{(i)}$ is the $i^{\text {th }}$ draw from $\chi_{T-1}^{2}$, Gelfand and Smith (1990), Casella and George (1992).

It is noted that $\frac{1}{L} \sum_{i=1}^{L} f\left(\hat{\beta} \mid w^{(i)}\right)$ converges to $\int_{0}^{\infty} f(\hat{\beta} \mid w) g(w) d w$ as $L \rightarrow \infty$, and the approximation is quite good for $L$ large enough. The theory behind the approximation (17) is the fact that the expected value of the conditional density $f(\hat{\beta} \mid W)$, when $W$ is a random variable, is

$E[f(\hat{\beta} \mid W)]=\int f(\hat{\beta} \mid w) g(w) d w=f(\hat{\beta})$

Thus, the formula in Equation (17) mimicks Equation (18), because $w_{1}^{(1)}, \ldots, w^{(L)}$ approximate a random sample from $g(w)$. Alternatively, we can think of Equation (18) as $E(X)=\mu$ where $\mu$ can be efficiently estimated by the sample mean $\bar{X}=\frac{1}{n} \sum_{i=1}^{n} X_{i}$, with $X_{1}, \ldots, X_{n}$ being a random sample from the distribution of $\mathrm{X}$. For large $n, \bar{X}$ converges to $\mu$. Similarly, for large $L, \frac{1}{L} \sum_{i=1}^{L} f\left(\hat{\beta} \mid w^{(i)}\right)$ converges to $\int f(\hat{\beta} \mid w) g(w) d w$, as claimed above. This is also called the Rao-Blackwellization and is quite popular in Markov chain Monte Carlo method, a recent fashion in Bayesian statistics. For more references, see Gilks et al. (1996).

The distribution of $\hat{\beta}$ is useful for testing hypothesis regarding $\beta$ because for any given $\beta$, the $100 \alpha \%$ value can be constructed as given in Karson et al. (1995). However, Karson et al. (1995) did not deal with the issue of the confidence interval of $\beta$ under asymmetric distribution of $\hat{\beta}$. This can be overcome by appealing to the asymptotic normal distribution of $\hat{\beta}$ as given below, 
February 2002 version

$\hat{\beta} \sim N\left(\beta, \sigma_{\hat{\beta}}^{2}\right)$,

where $\sigma_{\hat{\beta}}^{2}$ is given in (14).

One disadvantage of the asymptotic normal distribution for $\hat{\beta}$ is the symmetric assumption of the distribution of $\hat{\beta}$, although the exact distribution of $\hat{\beta}$ is not symmetric. A remedy of this problem is to consider the posterior distribution of $\beta$ using a Bayesian approach, which will lead to a natural posterior interval of $\beta$.

\section{A Bayesian approach for the inference of $\beta$}

In this section will consider the posterior distribution of $\beta$ using a noninformative prior distribution of $\mu$ and $\sigma^{2}$. Our ultimate goal is to contract a posterior interval of $\beta$. Let $Y_{1}, Y_{2}, \ldots \ldots Y_{\mathrm{T}}$ be i.i.d. $N\left(\mu, \sigma^{2}\right)$ and $\underset{\sim}{Y}=\left(Y_{1}, Y_{2}, \ldots \ldots, Y_{T}\right)$. The likelihood function of $\mu$ and $\sigma^{2}$ is:

$$
L\left(\mu,\left.\sigma^{2}\right|_{\sim} ^{Y}\right)=(2 \pi)^{-T / 2}\left(\sigma^{2}\right)^{-T / 2} e^{-\frac{1}{2 \sigma^{2}}\left\{(T-1) S^{2}+T(\mu-\bar{Y})^{2}\right\}} .
$$

Using the noninformative prior

$p\left(\mu, \sigma^{2}\right) \propto \frac{1}{\sigma^{2}}$,

and considering the transformations:

$\beta=\frac{\mu}{\sigma^{2}}+\frac{1}{2}$, and $\sigma^{2}=\sigma^{2}$,

we have the following posterior density of $\beta$ and $\sigma^{2}$ : 
February 2002 version

$$
\begin{aligned}
P\left(\beta, \sigma^{2} \mid Y\right) & \propto\left(\sigma^{2}\right)^{-\frac{T}{2}} e^{-\frac{(T-1) S^{2}}{2 \sigma^{2}}} e^{-\frac{T \sigma^{2}}{2}\left[\beta-\frac{1}{2}-\frac{\bar{Y}}{\sigma^{2}}\right]^{2}} \\
& =P\left(\beta \mid \sigma^{2}, \underset{\sim}{Y}\right) P\left(\left.\sigma^{2}\right|_{\sim}\right),
\end{aligned}
$$

where $\beta \mid \sigma^{2}, \underset{\sim}{\sim} \sim N\left(\frac{Y}{\sigma^{2}}+\frac{1}{2}, \frac{1}{T \sigma^{2}}\right)$, and $\theta=\frac{(T-1) S^{2}}{\sigma^{2}} \sim \chi_{T-1}^{2}$.

Thus,

$$
P(\beta \mid Y)=\int_{0}^{\infty} P(\beta \mid \theta, Y) P(\theta \mid Y) d \theta
$$

where

$$
\beta \theta, Y \sim N\left(\frac{\bar{Y} \theta}{(T-1) S^{2}}+\frac{1}{2}, \frac{\theta}{T(T-1) S^{2}}\right),
$$

and

$\theta \mid Y \sim \chi_{T-1}^{2}$.

The first two posterior moments of $\beta$ can be expressed as follows:

$$
\begin{aligned}
E(\beta \mid Y) & =\frac{\bar{Y}}{S^{2}}+\frac{1}{2} \\
V(\beta \mid Y) & =\operatorname{VVar}(\beta \mid \sigma, \underset{\sim}{Y})+\operatorname{VarE}(\beta \mid \sigma, \underset{\sim}{Y}) \\
& =\frac{T-1}{T(T-1) S^{2}}+\left(\frac{\bar{Y}}{(T-1) S^{2}}\right)^{2} 2(T-1) \\
& =\frac{1}{S^{2}}\left[\frac{1}{T}+\frac{2 \bar{Y}^{2}}{(T-1) S^{2}}\right] .
\end{aligned}
$$

This can be compared with $\operatorname{Var}(\hat{\beta})$ given in (17). 
As for the distribution of $\beta$, the posterior distribution of $\beta$, as given in Equation (21), can be approximated by

$P(\beta \mid Y \sim) \approx \frac{1}{L} \sum_{i=1}^{L} P\left(\beta \mid \theta^{(i)}, Y\right)$

where $\theta^{(i)}$ is the $i$ th draw of $\chi_{T-1}^{2}$.

Thus, an approximate $1-\alpha$ posterior interval $(a, b)$ of $\beta$ can be constructed from

$$
\int_{a}^{b} P(\beta \mid Y) d \beta=1-\alpha .
$$

It is noted that equal tail probability can be used in selecting $a$ and $b$, i.e., $a$ and $b$ can be selected such that both tail probabilities are $\frac{\alpha}{2}$. A better result is possible if we use the highest probability density (HPD) interval $\left(a^{*}, b^{*}\right)$ to insure the shortest posterior interval. However, if the posterior distribution of $\beta$ is nearly symmetric, as it is the case here, the construction of the HPD interval $\left(a^{*}, b^{*}\right)$ is not highly recommended.

\section{E. Empirical result}

In this section, we will use actual data to demonstrate how the new methods developed in previous sections can be used in empirical research. First, we demonstrate the advantage of the approximate method defined in Equation (17). Then, we test the normality of the data and propose Box-Cox transformation to check the validity of lognormality for the data. We also estimate the RRA values using the transformed data. Finally, we use the Bayesian method proposed in Section D to do the interval estimate for RRA.

Instead of using nonparametric approach adopted by Brown and Gibbons, we will propose the following Box-Cox transformation 


$$
X^{(\lambda)}=\left\{\begin{array}{cc}
\frac{X^{\lambda}-1}{\lambda}, & \text { if } \lambda \neq 0 \\
\ln X, & \text { if } \lambda=0
\end{array},\right.
$$

to examine whether the lognormal distribution for $X$ is suitable for the data at hand. If the power transformation parameter $\lambda$ is close to zero, then the lognormal distribution for $X$ is reasonable. Otherwise, the Box-Cox transformed variable $Y=X^{(\lambda)}$ is used in estimating the relative risk aversion.

The likelihood function of $\mu, \sigma^{2}$ and $\lambda$ is

$$
L\left(\mu, \sigma^{2},\left.\lambda\right|_{\sim} ^{X}\right)=(2 \pi)^{-\frac{T}{2}}\left(\sigma^{2}\right)^{-\frac{T}{2}} \exp \left[-\frac{1}{2 \sigma^{2}} \sum_{j=1}^{T}\left(X_{j}^{(\lambda)}-\mu\right)^{2}\right] \cdot J
$$

where

$$
J=\prod_{j=1}^{T} X_{j}^{\lambda-1} .
$$

The likelihood ratio test for testing the lognormality for $X$ within the Box-Cox family can be based on $-2 \log \Lambda$, where $\Lambda$ is the likelihood ratio criterion,

$$
\Lambda=\frac{\sup _{\mu, \sigma^{2}} L\left(\mu, \sigma^{2}, \lambda=\left.0\right|_{\sim} ^{Y}\right)}{\sup _{\mu, \sigma^{2}, \lambda} L\left(\mu, \sigma^{2},\left.\lambda\right|_{\sim} ^{Y}\right)} .
$$

The null hypothesis that the lognormality for $X$ is rejected at the significance level $\alpha$ if $-2 \log \Lambda>\chi_{1}^{2}(\alpha)$, where $\chi_{1}^{2}(\alpha)$ is the upper $100 \alpha$ percentile of the Chi-Square distribution with one degree of freedom.

Before presenting the empirical results using actual data, we will first compare the performance of the Rao-Blackwellization method given in Equation (17) with the exact distribution given in Equation (9), by assuming $T=30, \sigma=1.5$ and $\beta=1$ and 1.5. The results from Equation (17) are shown in Figure 1 with L=100 by first sampling 100 values from $\chi_{29}^{2}$ and then obtain the approximate $f(\hat{\beta})$ using Equation (17). This new method is definitely much easier than the exact method, which will require the summation of infinite 
number of terms in the formula. It is clear that both sampling distributions are almost identical to those shown in Figure 1 of Karson et al. (1995).

We next conduct the empirical study by collecting both monthly value-weighted index and riskless rate during the period December 1925 through December 1999. Using this set of data we calculated $X_{t}=\frac{1+R_{M t}}{1+R_{f t}}$ for doing the empirical study. A summary of the study is given in Table 1.

To determine graphically whether $X_{\mathrm{t}}$ or its transformation is normally distributed, we first normalized the data and redefined the variable as $z_{\mathrm{t}}$. We used time series plot, histogram and $Q-Q$ normal plot to show the goodness of fit to the normality of untransformed, log-transformed and the Box-Cox transformed data.

Figures 2 - 4 show for the three sets of untransformed, log-transformed and the Box-Cox transformed data for the period January 1953 to June 1967. From these plots we see that the Box-Cox transformed data are closer to the normal distribution.

In addition to the graphical presentation of the goodness of fit to normality, we have also conducted formal normality test using the likelihood ratio test for testing $\mathrm{H}_{0}: \lambda=0$ and the K-S statistic. The MLE of $\lambda$ is $\hat{\lambda}=4.133$ and the likelihood ratio test rejects the null hypothesis $\mathrm{H}_{0}: \lambda=0$ at significance level $\alpha=0.05$. Also K-S p-values are 0.0496 for the lognormal transformation and 0.253 for the Box-Cox transformation. Thus, it appears that the Box-Cox transformation is slightly better than the log transformation for the data considered in the period.

In Figure 5, we have also plotted the sampling distribution of $\hat{\beta}$ and the posterior distribution of $\beta$ by equations (17) and (26), respectively. It is clear that both distributions are quite symmetric and centered around 9.3. Also, the posterior distribution of $\beta$ is tighter than the sampling distribution of $\hat{\beta}$.

Comparable plots for the period July 1942 to December 1999 and the period January 1942 to December 1999 (annual December data) are given in Figures 6 - 9 and Figures $10-13$, respectively. In the period July 1942 to December 1999, the MLE of $\lambda$ is $\hat{\lambda}=3.705$ and the likelihood ratio test rejects $\mathrm{H}_{0}: \lambda=0$ at the significance level $\alpha=0.05$. 
Furthermore, the K-S p-values are 0.0002 and 0.584 for the log transformation and the Box-Cox transformation, respectively. Coupled with the accompanied plots, there are very strong evidences that the Box-Cox transformation is much better than the log transformation for the data in the period.

For the annual December data during the period January 1942 to December 1999, the MLE of $\lambda$ is $\hat{\lambda}=0.0099$ and the likelihood ratio test does not reject $\mathrm{H}_{0}: \lambda=0$ at the significance level $\alpha=0.05$. The K-S statistic is not computed because the sample size $T=58$ is rather small. A special note for this period is the plot in Figure 13. The mode of the sampling distribution of $\hat{\beta}$ is 16.82 while the medium is 18.45 , indicating that the sampling distribution of $\hat{\beta}$ is not symmetric. Meanwhile, the mode and medium of the posterior distribution of $\beta$ are 19.25 and 19.35, indicating that the posterior distribution is nearly symmetric. For this data set, we also found that the length of the $95 \%$ confidence interval for $\beta$ is 23.14 while the length of the posterior interval for $\beta$ is 21.08 , which is much shorter. Furthermore, the coverage probability for the Bayesian interval is 0.946 while the coverage probability for the confidence interval is 0.974 with $1-\alpha=0.95$. Thus, the Bayesian interval is shorter and with more accurate coverage probability.

\section{F.Summary}

In this paper, we first examine the validity of the lognormal distribution for the excess market rate of return. We then derived an alternative expression for the exact sampling distribution of the relative risk aversion estimator, which was obtained by Karson et al. (1995) in a different manner. An excellent approximation derived from the new expression is much easier to execute in obtaining the corresponding cumulative distribution function which is important in testing hypothesis regarding the relative risk aversion. Finally, we derived the posterior distribution of the relative risk aversion based on noninformative prior. The posterior distribution of the relative risk aversion enables us to construct posterior interval for the relative risk aversion, 
February 2002 version

which is shorter and with more accurate coverage probability than the corresponding confidence interval based on the asymptotic distribution of the relative risk aversion estimator. 


\section{References}

1. Box, G. E. P., and D. R. Cox, "An analysis of transformations," Journal of the Royal Statistical Society. Series B (Methodological), Vol. 26, No. 2. (1964), pp. 211-252.

2. Brown, D. P., and M. R. Gibbons, “A simple econometric approach for utilitybased asset pricing model," Journal of Finance, Vol. 40, No. 2, June 1985, pp. $359-381$.

3. Casella, G., and George, E. I., "Explaining the Gibbs sampler," American Statistician, Vol. 46, 1992, pp. 167- 174.

4. Gelfand, A. E., and A. F. M. Smith, "Sampling-based approaches to calculating marginal densities," Journal of American Statistics Association, Vol. 85, 1990, pp. $398-409$.

5. Gilks, W. R., S. Richardson, and Spiegelhalter, D. J., "Markov chain Monte Carlo in practice," 1996, Campman and Hall, London.

6. Karson, M., D. Cheng, and Lee, C., "Sampling distribution of the relative risk aversion estimator: theory and applications," Review of Quantitative Finance and Accounting, Vol. 5, No.1, March 1995, pp. 43 - 54.

7. Ljung, G. M., and G. E. P. Box, "Analysis of variance with autocorrelated observations," Scan. Journal of Statistics, Vol. 7, 1980, pp. $172-180$. 
February 2002 version

Table 1

Empirical Results on Excess Returns with Value-Weighted Indexes

\begin{tabular}{ccccccc}
\hline $\begin{array}{c}\text { Period } \\
\text { (Observation) }\end{array}$ & $\hat{\lambda}$ & LRT & Log & B-C & Mode & Median \\
\hline $\begin{array}{c}1 / 53-6 / 67 \\
(174)\end{array}$ & 4.133 & $\mathrm{R}$ & 0.0496 & 0.253 & & \\
\hline $\begin{array}{c}7 / 42-12 / 99 \\
(690)\end{array}$ & 3.705 & $\mathrm{R}$ & 0.0002 & 0.584 & & \\
\hline $\begin{array}{c}1 / 42-12 / 99 \\
\text { December }\end{array}$ & & & & & & \\
$(58)$ & 0.0099 & $\mathrm{~A}$ & & & 16.82 & 18.45 \\
\hline
\end{tabular}

* R for "Rejects" and A for "Accepts" the null hypothesis that $\lambda=0$ 